\title{
Estimation of Wind Penetration in a Thermal Dominated Power System Limited by Frequency Deviation
}

\author{
Yi-Zhen Wang*, Shi-Ying Ma, Qing Wang, Shi-Jun Ma, Yan-Long Sun \\ China Electric Power Research Institute, Beijing, 100192,China
}

\begin{abstract}
This paper describes a wind-thermal power system on the analysis of the frequency response characteristics of thermal generators and wind turbines (WTs) against the imbalanced power. The composite transfer function of the speed governing system is proposed on the basis of the speed governor's model, and the time-domain expression of the frequency deviation against the perturbation of electrical power is given in this paper. This paper studies the maximum surplus power $\left(P_{s, m}\right)$ and the maximum deficit power $\left(P_{d, m}\right)$ in a thermal dominated power system to avoid the action of OPC and UPLS. Considering the wind farm (WF) power injected to the existing power system, the wind penetration level $\left(L_{P}\right)$ has great effects on the $P_{s, m}$ and the $P_{d, m}$. Through theoretical calculation and the simulation of a power system that consists of a thermal unit and GE wind turbines, this paper draws this conclusion that with the increase of the wind penetration level, $P_{s, m}$ will increase in a liner relation and $P_{d}$ will decrease in a hyperbolic relation.
\end{abstract}

Keywords: Wind penetration level, OPC, UPLS, the maximum surplus power, the maximum deficit power, overshoot

\section{Introduction}

Reference [1]-[3] denote that the penetration level is conservatively estimated to be $50 \%$ for optimal wind power acquisition, reference [1] discusses the transfer function of the overall closed loop feedback of the speed-governor for the thermal generator, and analyzes the frequency response lessons from simulation experiments, reference [2] discusses the permitted power fluctuation [in per unit (p.u.)] for the $1 \%$ frequency deviation on the basis of reference [1], reference [3] discusses the Temporary MInimum Frequency (TMIF) in a hydro dominated power system, draw a conclusion that the bigger is the wind penetration level in a power system, the lower is TMIF in a power deficit situation, and wind turbines respond much quicker than conventional generators such as the hydro and the thermal units in a imbalanced power situation. The present works mainly discuss the TMIF in a power deficit scenario, few works discuss the TMAF in a power surplus scenario, in fact, the over-frequency problems in a thermal dominated power system are also of great importance (see [4]-[6]).

This paper is mainly divided into three major sections, Sections 2 mainly describes the frequency response characteristics of the thermal generator in a imbalanced power situation, Sections 3 mainly discusses the respond speed of the control system of WTs and its application to the frequency control system, Sections 4 discusses the impacts on the $P_{d, m}$ and $P_{s, m}$.

\section{Frequency Response Characteristics of Thermal Generators}

Fig. 1 is the complete model of speed governing system for a thermal unit, it involves three parts: the first one is the speed governor, the second one is the steam turbine, the third one is the generator (the

\footnotetext{
* Manuscript received June 12, 2012; revised July 6, 2012.

Corresponding author. Tel.: +86-13426427647; E-mail address: wangyizhen@epri.sgcc.com.cn.
} 
swing model), where $\Delta P_{E}$ is the electrical power, $\Delta P_{M}$ is the mechanical power, $R$ is the speed droop, $\mathrm{K}$ is the forward coefficient, $T_{s m}$ is the time constant of servomotor, $P_{\text {set }}$ is the reference of electrical power, $\Delta \omega$ is the perturbation of rotor speed of the generator, $\mathrm{H}$ is the inertia constant.

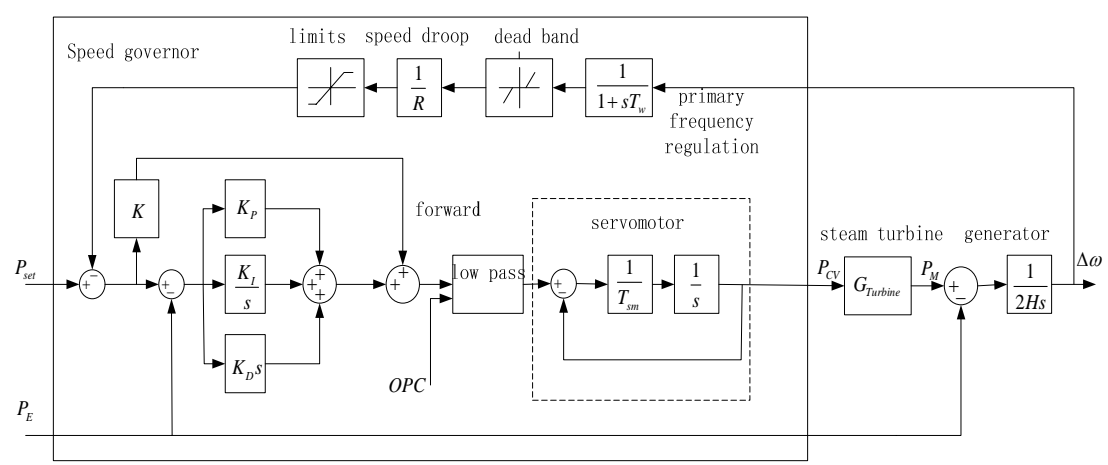

Fig. 1. Block diagram of complete model of speed governing system. Values of different parameters are given in the Appendix

\subsection{The OPC principle and the transfer function of the steam turbine}

The speed governor of thermal units usually has the OPC device to protect the generator itself, unfortunately, when the surplus power is large, OPC would operate repeatedly, resulting in the frequency and power fluctuation, and generators will quit finally (reference [4]). Fig. 2 shows when the surplus power is $35 \%$, OPC operates repeatedly, the mechanical power and rotor speed (frequency) fluctuates along the time.
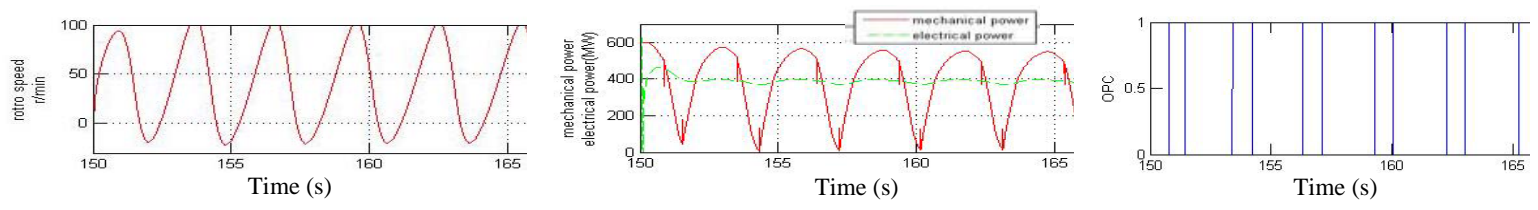

Fig. 2. Over-speed problems representation (the surplus power is 0.35 p.u).

The detailed model of steam turbine see reference [7], and the simplified transfer function of the turbine relating perturbed values of the mechanical power $\left(\Delta P_{M}\right)$ and control valve position $\left(\Delta P_{C V}\right)$ may be written as follows, where $F_{H P}$ is the fraction of total turbine power generated by HP, $\lambda$ is the natural power overshooting coefficient, $T_{C H}$ is the time constant of main inlet volumes and steam chest, $T_{R H}$ is the time constant of reheater.

$$
G_{\text {Turbine }}=\frac{\Delta P_{M}}{\Delta P_{C V}}=\frac{1+s(1+\lambda) F_{H P} T_{R H}}{\left(1+s T_{C H}\right)\left(1+s T_{R H}\right)}
$$

\subsection{The composite transfer function of the speed governing system}

This paper describes the frequency response characteristics against the perturbation of electrical power, using the Mason's Rule in reference [8] and the block diagram in Fig.1, the simplified transfer function of the generator relating perturbed values of rotor speed $(\Delta \omega)$ and the electrical power $\left(\Delta P_{E}\right) \&$ the reference of electrical power ( $\Delta P_{\text {set }}$ ) may be written as follows:

$$
\Delta \omega=\frac{\left(K+G_{P I D}\right) G_{\text {Turbine }}}{\left(s^{2} 2 H T_{\text {sm }}+s 2 H\right)+\left(K+G_{P I D}\right) G_{\text {Turbine }} K_{G}} \Delta P_{\text {set }}-\frac{G_{P I D} G_{\text {Turbine }}+s T_{s m}+1}{\left(s^{2} 2 H T_{s m}+s 2 H\right)+\left(K+G_{P I D}\right) G_{\text {Turbine }} K_{G}} \Delta P_{E}
$$

If there is no other measures to taken, i.e., the reference of electrical power doesn't change, $\Delta P_{\text {set }}=0$, equation (2) will be simplified as follows: 


$$
\frac{\Delta \omega}{\Delta P_{E}}=-\frac{G_{P I D} G_{\text {Turbine }}+s T_{\text {sm }}+1}{\left(s^{2} 2 H T_{s m}+s 2 H\right)+\left(K+G_{P I D}\right) G_{\text {Turbine }} K_{G}}
$$

For a thermal unit, the parameters in (3) are certain and clear, take one thermal unit (600MW, Reheat Turbine, see details in the Appendix) as an example, quantifies correlative parameters in (1) and (3):

$$
\begin{aligned}
G_{\text {Turbine }} & =\frac{1+5.4 s}{(1+0.18 s)(1+11.276 s)} \\
\frac{\Delta \omega}{\Delta P_{E}} & =\frac{0.4 s^{4}+4.32 s^{3}+18.136 s^{2}+2.56 s+0.0667}{3.45 s^{5}+36.64 s^{4}+100.6 s^{3}+206 s^{2}+43.44 s+1.48}
\end{aligned}
$$

Formula (5) is a high order homogeneous equation, has 4 zeros $(-5.327-3.925 i,-5.327+3.925 i,-0.11$, $-0.034)$ and 5 poles $(-1.27-2.279 i,-1.27+2.279 i,-0.1892,-0.0424,-7.848)$. According to Reference [8], these zeros and poles which absolute value is 6 times bigger than the real part of the main pole have little effect on the result, so formula (5) can be simplified as follows:

$$
\frac{\Delta \omega}{\Delta P_{E}}=\frac{k_{1} \times(s+0.11)(s+0.034)}{(s+0.1892)(s+0.0424)(s+7.848)}
$$

where $k_{1}$ is a constant that make the final value of formula (6) equal with the final value of formula (5), and in this case its value is 0.7683 , the step response of transfer function (6) is:

$$
\Delta \omega(s)=\frac{1}{s} \times \frac{k_{1} \times(s+0.11)(s+0.034)}{(s+0.1892)(s+0.0424)(s+7.848)}
$$

And its time-domain expression is:

$$
\Delta \omega(t)=\left(0.0117 e^{-0.424 t}-0.1289 e^{-7.848 t}+0.0578 e^{-0.1892 t}+0.0594\right) \times 0.7683
$$

Through calculation, the maximum value of formula (8) is 0.0932 at $t_{p}=0.584 \mathrm{~s}$, so the maximum overshoot is

$$
\sigma \%=\frac{\Delta \omega_{\max }-\Delta \omega_{\infty}}{\Delta \omega_{\infty}} \times 100 \%=\frac{0.0932-0.0451}{0.0451} \times 100 \%=100 \%
$$

\subsection{The maximum surplus power and the maximum deficit power}

According to 3.2, the temporary maximum/minimum frequency (TMAF/TMIF) will occur at about $0.584 \mathrm{~s}$ after detecting the disturbance, the maximum frequency deviation is about two times of the final frequency deviation.

For example, in China, TMIF $>49 \mathrm{~Hz}$ (to avoid the operation of UFLS), TMAF $<51.5 \mathrm{~Hz}$ (to avoid the operation of OPC), $P_{s, m}$ must satisfy the following equation:

$$
P_{s, m} / P_{E}=K_{G} \times \Delta \omega_{\infty}=K_{G} \times \frac{\Delta \omega_{\max }}{1+\sigma \%}
$$

Take the example in section 3.1 again, quantifies $P_{s, m}$ using the real parameters in the Appendix, $P_{s, m}=33 \%$. Because $P_{s}=35 \%>P_{s, m}=33 \%$ in section 3.1 example, OPC operate repeatedly as shown in Fig.2. Reconsidering this example but $P_{s}=30 \%$, the result is shown in Fig.3, frequency hasn't reached $51.5 \mathrm{~Hz}$, OPC doesn't act.

In the same way, this paper indicates the deficit power must below $P_{d, m}=22 \%$ to prevent the UFLS operating in the same example using parameters in the Appendix. 


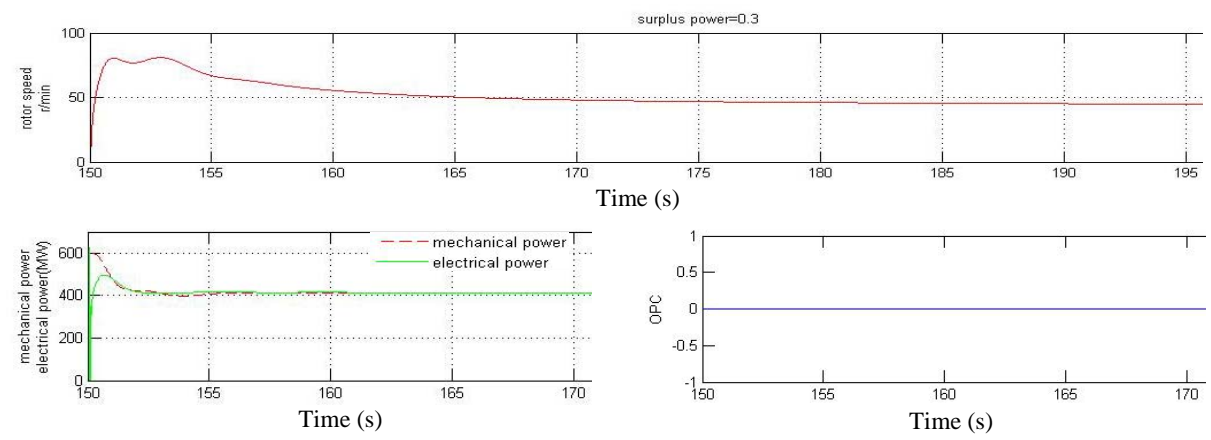

Fig. 3. Over-speed problems representation (the surplus power is $0.3 \mathrm{p.u}$ ).

\section{The control system of wind turbines}

Before the late 1990s, most wind generator manufacturers around the world built fixed-speed wind plant system (FSWPS) using a gearbox and a standard squirrel-cage induction generator, directly connected to the grids [9]. Since the late 1990s, most wind plant manufacturers have changed to variable speed wind turbines (VSWTs) [10]. Many researches have been done to study the detailed model of all kinds of wind turbines. This paper just focuses on the response speed of the control system of the pitchcontrolled WTs and the impact on the system inertia with the increase of the wind penetration level in a thermal dominated power system.

\subsection{The control system of WTs}

A published model of a multi-megawatt commercial pitch-controlled WTs (GE 1.5MW) is used in this work which is adopted from [11]. The block diagram of the wind turbine model is shown in Fig.4.

The aerodynamic part can be modeled by equation (11):

$$
P_{m}=0.5 \rho A C_{p}(\lambda, \theta) v_{w}^{3}
$$

Where $P_{m}$ is mechanical power captured by turbine, $\rho$ is air density, A is swept area by blades, $C_{P}$ is power coefficient, $v_{w}$ is wind velocity and $\theta$ is pitch angle. $\lambda$ is tip speed ratio (TSR) and can be defined by $\lambda=R \omega / v_{w}, C_{P}-\lambda, \theta$ equation is given in the Appendix.

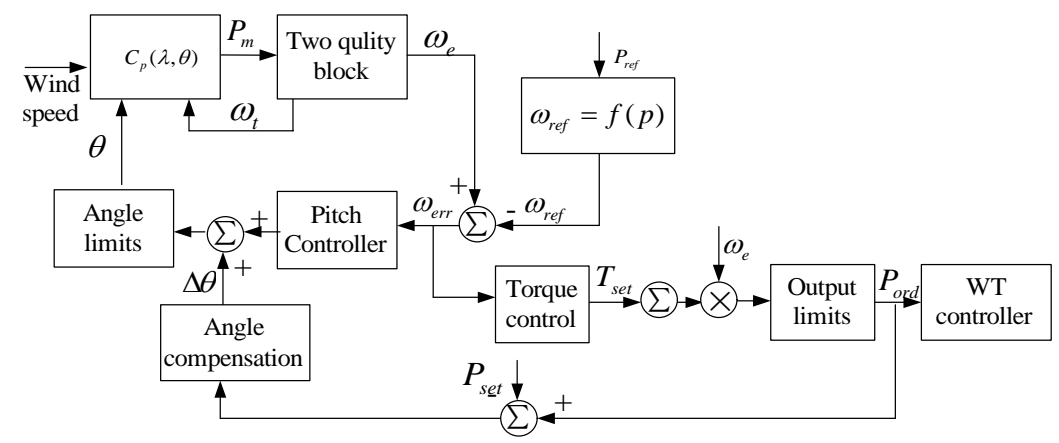

Fig. 4. Block diagram of a wind turbine controller model. Values of different parameters are given in the Appendix.

\subsection{A new strategy to control frequency deviation using pitch angle $\theta$}

The response speed of pitch-controlled WTs to the imbalanced power becomes more and more important as the wind penetration level increases, the mechanical power $\mathrm{P}_{\mathrm{m}}$ is in proportion to value of $\mathrm{C}_{\mathrm{p}}$ from formula (11), and there is a liner relationship between $\mathrm{C}_{\mathrm{p}}$ and the pitch angle $\theta$ from the Appendix, so a new strategy to control the imbalance power using $\theta$ is proposed in this paper: 
Suppose that the wind speed and the rotor speed haven't change in arresting the initial frequency increase, the mechanical power is:

$$
\begin{aligned}
& P_{m}=0.5 \rho A C_{p}(\lambda, \theta) v_{w}^{3}=0.5^{2} \rho A v_{w}^{3}\left(v_{p}-0.022 \theta-2\right) \exp \left(-0.255 v_{p}\right) \\
& v_{p}=C_{f} v_{w} / \omega
\end{aligned}
$$

The pitch angle $\theta$ could be changed quickly by adjusting the PI controller parameters in the pitch controller model, so the mechanical power can be changed quickly in pitch-controlled WTs compared with the conventional generators, especially when pitch-controlled WTs work on the best operating point with $\theta=0$ in the power surplus scenario. This means pitch-controlled WTs could change the output in a very short time, and the application of this characteristic could be discussed in section $\mathrm{V}$ in detail.

\section{The impact on $\boldsymbol{P}_{d, m}$ and $\boldsymbol{P}_{s, m}$ wind penetration brings}

According to section IV, pitch-controlled WTs could change the output in a very short time compared with the conventional generators, reference [3] and [12] also denotes that pitched-controlled WTs could support the frequency by changing the output of WTs before the speed governor taking actions in the conventional generators, those WTs that can not control pitch angle could quit from the power system when detecting the initial frequency increase.

The work is done based on the following assumptions:

- The total load of the system remains the same.

- The wind turbine to be installed does not respond to the network frequency deviation, i.e., no network frequency sensitive extra active power support from the wind turbines.

- The droop setting of the individual generators remain the same, i.e., an increase in the equivalent droop (decrease in the equivalent gain) with increasing wind penetration level.

- An $L_{p}$ wind penetration means an $L_{P}$ reduction in the existing generating units, i.e., an $L_{P}$ reduction in the system inertia. The steady load balance is maintained by the $L_{P}$ wind generation.

\subsection{Impact on $P_{s, m}$}

As shown in Fig. 5, the support from the WFs can be added to the imbalanced power and the combined effect is a reduction in the surplus power. $\Delta P_{W}$ is the decreased power that WFs supports in the first few seconds before the speed governor in the thermal generator acts, $\Delta P_{W}$ is multiplied with the penetration level $L_{P}$ to convert $\Delta P_{E}$ from the WF base to the system MVA base. The effective perturbation electrical power in the presence of the WFs is

$$
\Delta P_{E, \text { new }}=\Delta P_{E}-\Delta P_{W} L_{P}
$$

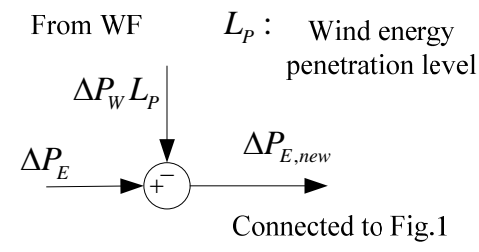

Fig. 5. Augmentation of the WT into the existing thermal power system

With this new perturbation electrical power, the new $P_{s, m}$ can be calculated from (10). When the power surplus scenario happens (including the load drop off and the tie line between the thermal dominated power system and the load centre disconnects suddenly), the WFs can decrease the electrical power output in a very short time to protect the thermal generators because it is harder in technology and more expensive in economic to restart a thermal generator and re-connect it to the power system. In the extremist cases, the WFs shut down when the surplus is too large, through calculation from (10) and (14), 
$P_{s, m}$ increases as the wind penetration increases in a liner relation:

$$
P_{s, \text { new }}=P_{s, \text { old }}+L_{p}
$$

Add 20 GE 1.5MW wind turbines to the existing thermal dominated power system (example in section III), i.e., the wind penetration level $L_{P}=5 \%$, when $P_{s}=35 \%$, the result is shown in Fig.6, the fourth column shows the output of the WF decreases to zero abruptly after power surplus scenario happens, so the imbalance power decreases to such a acceptable level that OPC in the speed governor of the thermal generator will not operate. Comparing Fig. 6 with Fig. 3 illustrates the impact on $P_{s, m}$ wind penetration brings.
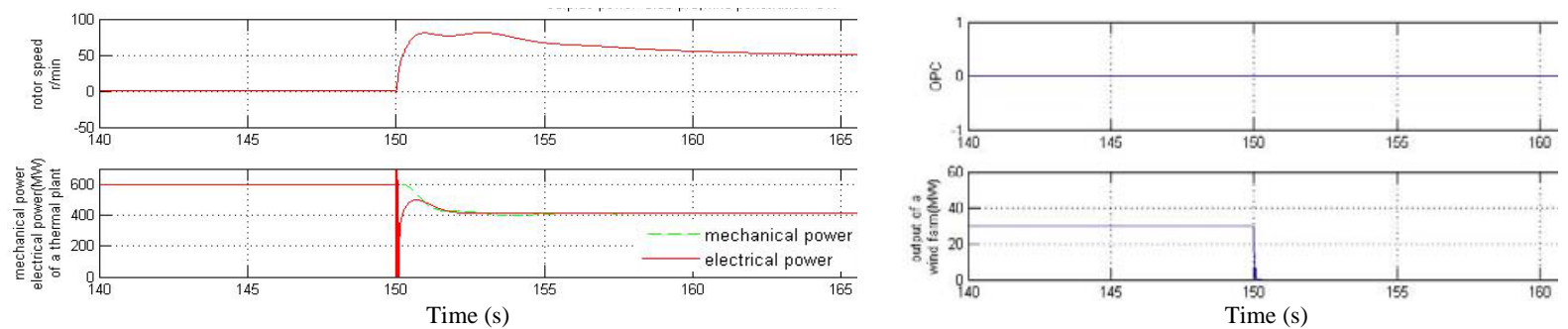

Fig. 6. Over-speed problems representation (the surplus power is 0.35 p.u and the $\mathrm{Lp}=5 \%$ )

\subsection{The impact on $P_{d, m}$}

Operating a large number of WTs displaces conventional synchronous generators and reduces the system inertia, this is undesirable when wind penetration increases, especially in periods of low load and on smaller power systems [13].

When the wind penetration level is $L_{P}$, the system inertia constant becomes $H\left(1-L_{P}\right)$, and the droop becomes $R /\left(1-L_{P}\right)$, so the formula (3) becomes as follows:

$$
\frac{\Delta \omega}{\Delta P_{E}}=-\frac{G_{P I D} G_{\text {Turbine }}+s T_{s m}+1}{\left(\left(s^{2} 2 H T_{s m}+s 2 H\right)+\left(K+G_{P I D}\right) G_{\text {Turbine }} K_{G}\right)\left(1-L_{P}\right)}
$$

so the maximum overshoot is

$$
\sigma \%_{e q}=\sigma /\left(1-L_{P}\right) \%
$$

The temporary minimum frequency $\left(T M I F_{e q}\right)$ becomes as follows:

$$
\mathrm{TMIF}_{\text {eq }}=50-(50-\mathrm{TMIF}) /\left(1-L_{P}\right)
$$

Reconsider the maximum deficit power $\mathrm{P}_{\mathrm{d}}$,

$$
P_{d, m} / P_{E}=K_{G} \times \Delta \omega_{\infty}=K_{G} \times \frac{\Delta \omega_{\max }}{1+\sigma_{e q} \%}=K_{G} \times \frac{\Delta \omega_{\max }\left(1-L_{P}\right)}{1-L_{P}+\sigma \%}
$$

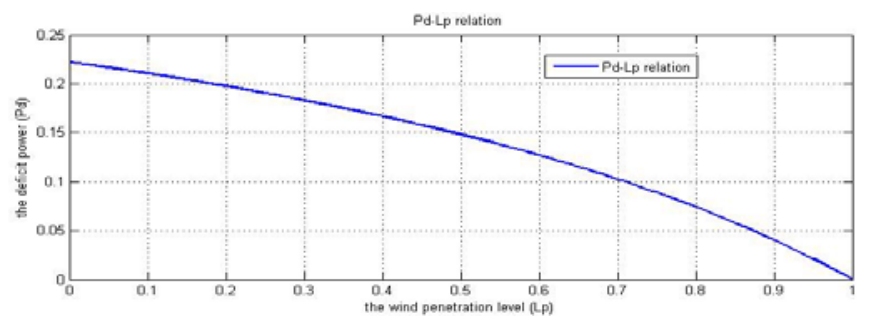

Fig. 7. Relation between $P_{d}$ and $L_{P}$

Take the former example (in section 5.1) again, the relation between $P_{d}$ and $L_{P}$ is shown in Fig.7, when $P_{L}=0, P_{d, m}=22 \%$ (have discussed in section 3.3), when $P_{L}=10 \%, P_{d, m}=21 \%$, and the deficit power decrease relative slow as the wind penetration level increase from 0 to $30 \%$. 


\section{Conclusion}

The frequency response characteristic of thermal generators is described by using the composite transfer function of a small system of thermal power generators. In order to coordinate with OPC of a thermal generator and the UFLS settings, this paper quantifies the maximum mismatched power in a thermal dominated power system. Take a thermal unit as an example (see the Appendix), the maximum deficit power is $22 \%$ and the maximum surplus power is $33 \%$ in China.

Along with the wind penetration increases, the maximum surplus power $P_{s}$ will increase in a liner relation while the maximum deficit power $P_{d}$ will decrease in a hyperbolic relation, so $P_{s}$ and $P_{d}$ is in a hyperbolic relation as the wind penetration increases.

\section{References}

[1] Luo C, Ooi BT. Frequency deviation of thermal power generators due to wind farms. IEEE Trans. on Energy Conversation; 2006; 21(3): 708-716.

[2] Luo C., Far HG, Banakar H, et al. Estimation of wind penetration as limited by frequency deviation. IEEE Trans. on Energy Conversation, 2007; 22(3):783-791.

[3] Ullah NR, Thiringer T, Karlsson D. Temporary primary frequency control support by varible speed wind turbines-potential and applications. IEEE Trans. on Power Systems, 2008; 3(2):601-612.

[4] Huang ZJ, Chao J, Lu XY, et al. High frequency phenomenon caused by faults occurred in southern part of Guiyang power grid and simulation of overspeed protection controller, Power System Technology, 2007; 31(15):26-32.

[5] Deng J, Li XY, Wei W. OPC controller for turbo-generating set based on immune fuzzy algorithm. Proceedings of the CSUEPSA, 2011; 23(3):1-7.

[6] Huang ZJ, LI XY, Chao J, et al. Guiyang south grid '7.7' faults simulation and analysis. Automation of Electric Power System; 2007; 31(9):95-100.

[7] Tian YF, Guo JY, Liu YQ, et al. A mathematical model of reheat turbine for power grid stability calculation. Power System Technology, 2007; 31(5):39-44.

[8] Hu SS. Principle of Automatic Control. 4rd ed. Beijing: Science; 2001.

[9] Sloothwge JG, De Vries E. Inside wind turbine-fixed vs variable speed. Renew Energy World, 2003; 6(1):30-40.

[10] Polinder H, De Haan SWH, Sloothwge JG, et al. Basic operation principles and electrical conversion systems of wind turbines. EPE Journal, 2005; 15(4):43-50.

[11] Miller NW, Price WW, Sanchez-Gasca JJ. Dynamic modeling of GE 1.5 and 3.6 wind Turbine-Generators. In: GE-Power System Energy Consulting, Tech. Rep. Version 3.0, Oct. 2003.

[12] Moutis P, Papathanasiou S, Hatziargyriou ND. Primary load-frequency control from pitch-controlled wind turbines. In: Proc. of IEEE Bucharest Power Tech Conference, 2009:1-7

[13] Ekanayake J, Jenkins N. Comparison of the response of doubly fed and fixed-speed induction generator wind turbines to changes in network frequency. IEEE Trans. on Energy Convesion, 2004; 19(4):800-802.

\section{An example appendix}

\section{A.1. Example of a thermal unit}

China Production, $600 \mathrm{MW}$, single reheat, tandem-compound, parameters are as follows:

Table 1. parameters of a thermal unit

\begin{tabular}{ccccccccccccc}
\hline Capacity(MVA) & $F_{H P}$ & $F_{I P}$ & $F_{L P}$ & $\lambda$ & $T_{C H}(\mathrm{~s})$ & $T_{R H}(\mathrm{~s})$ & $T_{S M}(\mathrm{~s})$ & $K_{G}$ & $K$ & $K_{P}$ & $K_{I}$ & $H(\mathrm{~s})$ \\
\hline 600 & 0.3 & 0.3 & 0.4 & 0.6 & 0.1812 & 11.276 & 0.2 & 22.22 & 0.35 & 1.2 & $1 / 15$ & 4.232 \\
\hline
\end{tabular}

A.2. $C_{p}-\lambda, \theta$

The approximate formula for $C_{p}$ is as belows: 


$$
C_{p}=0.5\left(\frac{R C_{f}}{\lambda}-0.022 \theta-2\right) \exp \left(-0.225 \frac{R C_{f}}{\lambda}\right)
$$

where $C_{f}$ is the design constant of the blade, usually $1 \sim 3$.

\section{A.3. Example of a wind turbine}

WT physical parameters: $H_{W T}=5.19 \mathrm{~s}, 0.5 \rho A / S_{n}=0.00145, R=52 \mathrm{~m}$.

WT control parameters: $V_{W T}=1.0$ p.u, pitch controller-(150+(25/s)). 